\title{
High-resolution MEGARA Integral-field Unit Spectroscopy and Structural Analysis of a Fast-rotating, Disky Bulge in NGC 7025
}

\author{
Bililign T. Dullo ${ }^{1}$ (10), Mario Chamorro-Cazorla ${ }^{1}$, Armando Gil de Paz ${ }^{1}$ (D) África Castillo-Morales ${ }^{1}$ (D) , Jesús Gallego ${ }^{1}$ (D), \\ Esperanza Carrasco ${ }^{2}$, Jorge Iglesias-Páramo ${ }^{3,4}$ (i), Raquel Cedazo ${ }^{5}$, María Luisa García-Vargas ${ }^{6}$, Sergio Pascual ${ }^{1}$ (i), \\ Nicolás Cardiel ${ }^{1}$ (D), Ana Pérez-Calpena ${ }^{5}$, Pedro Gómez-Alvarez ${ }^{5}$, Ismael Martínez-Delgado ${ }^{6}$, and Cristina Catalán-Torrecilla ${ }^{1}$ (iD \\ ${ }^{1}$ Departamento de Física de la Tierra y Astrofísica, Instituto de Física de Partículas y del Cosmos IPARCOS, Universidad Complutense de Madrid, \\ E-28040 Madrid, Spain; bdullo@ucm.es \\ ${ }^{2}$ Instituto Nacional de Astrofísica, Óptica y Electrónica, Luis Enrique Erro No. 1, C.P. 72840, Tonantzintla, Puebla, Mexico \\ ${ }^{3}$ Instituto de Astrofísica de Andalucía-CSIC, Glorieta de la Astronomía s/n, E-18008, Granada, Spain \\ ${ }^{4}$ Estación Experimental de Zonas Áridas - CSIC, Ctra. de Sacramento s/n, 04120 Almería, Spain \\ ${ }^{5}$ Universidad Politécnica de Madrid, Madrid, Spain \\ ${ }^{6}$ FRACTAL S.L.N.E. C/ Tulipán 2, p13, 1A. E-28231, Las Rozas de Madrid, Spain \\ Received 2018 August 4; revised 2018 November 24; accepted 2018 November 25; published 2019 January 17
}

\begin{abstract}
Disky bulges in spiral galaxies are commonly thought to form out of disk materials (mainly) via bar-driven secular processes. They are structurally and dynamically distinct from "classical bulges," which are built in violent merger events. We use high-resolution GTC/MEGARA integral-field unit spectroscopic observations of the Sa galaxy NGC 7025, obtained during the MEGARA commissioning run, together with detailed 1D and 2D decompositions of this galaxy's Sloan Digital Sky Survey $i$-band data to investigate the formation of its disky (bulge) component, which makes up $\sim 30 \%$ of the total galaxy light. With a Sérsic index $n \sim 1.80 \pm 0.24$, a half-light radius $R_{\mathrm{e}} \sim 1.70 \pm 0.43 \mathrm{kpc}$, and stellar mass $M_{*} \sim(4.34 \pm 1.70) \times 10^{10} M_{\odot}$, this bulge dominates the galaxy light distribution in the inner $R \sim 15^{\prime \prime}(\sim 4.7 \mathrm{kpc})$. Measuring the spins $(\lambda)$ and ellipticities $(\epsilon)$ enclosed within nine different circular apertures with radii $R \leqslant R_{\mathrm{e}}$, we show that the bulge, which exhibits a spin track of outwardly rising $\lambda$ and $\epsilon$, is a fast rotator for all the apertures considered. Our findings suggest that this inner disky component is a pseudo-bulge, consistent with the stellar and dust spiral patterns seen in the galaxy down to the innermost regions but in contrast to the classical bulge interpretation favored in the past. We propose that a secular process involving the tightly wound stellar spiral arms of NGC 7025 may be driving gas and stars out of the disk into the inner regions of the galaxy, building up the massive pseudo-bulge.
\end{abstract}

Key words: galaxies: bulges - galaxies: elliptical and lenticular, cD - galaxies: kinematics and dynamics galaxies: photometry - galaxies: spiral - galaxies: structure

\section{Introduction}

A large number $(\sim 70 \%)$ of observed spiral and S0 galaxies contain a bulge component (e.g., Gavazzi et al. 2000, their Figure 13; Böker et al. 2003; Allen et al. 2006; Cameron et al. 2009). Excluding the innermost regions, bulges are typically evident by the dominance of the inner stellar light distribution of their host galaxies with respect to the outer disk's light profile. Earlier models of galaxy formation predicted that present-day bulges and elliptical galaxies are pressuresupported systems with old stellar populations, described by the $R^{1 / 4}$ law (de Vaucouleurs 1948) and formed hierarchically through mergers of smaller systems (e.g., Barnes 1988; Kauffmann 1996) or built in the monolithic collapse of protogalactic gas clouds (Eggen et al. 1962). Confirming the existence of such pressure-supported elliptical "classical bulges" generally well described by the Sérsic (1968) $R^{1 / n}$ model, subsequent studies have revealed disklike "pseudobulges,"6 which show significant rotational support and tend to have a range of ages and low $n(\lesssim 2)$ Sérsic light profiles (e.g., Kormendy 1982, 1993; Kormendy \& Illingworth 1982; Pfenniger 1993; Courteau et al. 1996; Carollo et al. 1997;

\footnotetext{
6 Throughout this paper, we use the term "pseudo-bulge" when referring to "disklike" bulges, although this term is also sometimes used to refer to "boxy/ peanut-shaped" bulges, which are now understood to be thick bars seen edgeon (e.g., Combes et al. 1990; Kuijken \& Merrifield 1995; Bureau \& Freeman 1999; Athanassoula 2005; Laurikainen \& Salo 2016).
}

Kormendy \& Kennicutt 2004; Athanassoula 2005; Laurikainen et al. 2007; Fisher \& Drory 2008; Graham \& Worley 2008; Gadotti 2009; Kormendy 2013; Erwin et al. 2015; Tonini et al. 2016; Catalán-Torrecilla et al. 2017). The difference between classical bulges and pseudo-bulges is thought to reflect two distinct bulge formation paths, although both types of bulges can coexist in a galaxy (Erwin et al. 2003; Athanassoula 2005; Peletier 2008; Erwin et al. 2015; Dullo et al. 2016).

Pseudo-bulges are largely believed to be formed out of disks via secular evolution driven by non-axisymmetric stellar structures, such as bars. However, little is known about the formation of pseudo-bulges in unbarred disk galaxies, which account for roughly $30 \%$ of local disk galaxies (de Vaucouleurs 1963; Knapen et al. 2000; Sheth et al. 2008; Buta et al. 2015; Erwin 2018). Alternative pseudo-bulge formation channels have recently been discussed in the literature. For example, pseudo-bulges have been suggested to be built through gas-rich minor and/or major galaxy merger events (e.g., Springel \& Hernquist 2005; Eliche-Moral et al. 2011; Guedes et al. 2013; Querejeta et al. 2015; Athanassoula et al. 2016; Sauvaget et al. 2018) or formed at a high redshift via starbursts (Okamoto 2013) or through clumps that sink to the galaxy center by dynamical friction (Inoue \& Saitoh 2012).

Detailed structural and stellar kinematic studies of pseudobulges enable us to discriminate between these different formation scenarios, but the lack of robust bulge diagnostic 
criteria presents a major challenge for the identification of pseudo-bulges (e.g., Graham 2013; Neumann et al. 2017). Graham (2013, his Section 4.3) provided cautionary remarks about the misidentification of pseudo-bulges and classical bulges when using criteria based, for example, on the bulge's Sérsic index, rotation, and stellar age (Kormendy \& Kennicutt 2004, their Section 4; Fisher \& Drory 2008). A related issue in the structural analysis of bulges is the failure to account for small- and intermediate-scale components when modeling the stellar light distributions of disk galaxies. Dullo et al. (2016, 2017, 2018; see also Graham et al. 2016) showed that neglecting to fit components such as bars, disks, and spiral arms as a separate component can systematically bias the Sérsic index and flux of the bulge component toward higher values (e.g., de Jong 1996; Laurikainen et al. 2005; Gadotti 2008).

Here we investigate the bulge of the spiral (Sa) galaxy NGC 7025 (RC3; de Vaucouleurs et al. 1991). This galaxy is a promising candidate to explore the agents that drive the buildup of pseudo-bulges out of disk materials via secular evolution in unbarred galaxies. It is the only unbarred isolated galaxy in the sample of 49 CALIFA galaxies by Holmes et al. (2015) which displays noncircular, barlike flows. The galaxy is among the first galaxies observed with the MEGARA integral-field unit (IFU) as part of the commissioning run of the MEGARA instrument. The CALIFA kinematic maps for the galaxy by Falcón-Barroso et al. (2017, their Figures A.1 and A.2), extracted using the medium-resolution CALIFA data, have a large field of view which extends to the outer disk regions of the galaxy. These maps complement our MEGARA data, which have higher spatial and spectral resolutions and cover the inner half-light radius of the bulge. For comparison, the medium-resolution CALIFA data have a spectral resolution $R_{\text {spec }} \sim 1650$ at $\sim 4500 \AA$ (Falcón-Barroso et al. 2017), while the lowest spectral resolution of MEGARA is $R_{\text {spec }} \sim 6000$.

Our isophotal analysis of the Sloan Digital Sky Survey (SDSS) images of NGC 7025 reveals a "disky" bulge. Recently, Neumann et al. (2017), Rizzo et al. (2018), and Gilhuly \& Courteau (2018) fit a 2D Sérsic bulge + exponential disk model to the galaxy's SDSS images. Neumann et al. (2017) argued that the galaxy has a merger-built classical bulge with a bulge-to-total flux ratio $(B / T) \sim 0.44$, consistent with the fit by Gilhuly \& Courteau (2018), which yielded a high Sérsic index for the bulge ( $n \sim 5.1$ ), suggestive of a classical bulge. In contrast, the bulge + disk fit by Rizzo et al. (2018) yielded a lower value of $n(\sim 2.4)$ and a lower $B / T$ of $\sim 0.3$ for the bulge component.

High-resolution structural and stellar kinematic studies of the bulges of unbarred spiral galaxies such as NGC 7025 are of interest because they can provide clues to the processes of galaxy formation and evolution. For the first time, we perform $1 \mathrm{D}$ and 2D four-component (i.e., point source + bulge + intermediate-scale spiral-arm component + outer disk) fits to the SDSS data of NGC 7025. Combining these fits together with the analyses of the galaxy images and our new highresolution MEGARA IFU spectroscopic observations, we favor a pseudo-bulge interpretation for the bulge, which is built via secular evolution driven by the spiral arms in the galaxy. Introducing the MEGARA instrument in Section 2.1.1, we go on to describe our MEGARA spectroscopic observations of the galaxy and the subsequent data reduction steps in Sections 2.1.2 and 2.1.3, respectively. The SDSS data for the galaxy and the corresponding data reduction steps are detailed in Section 2.2. Our structural decompositions, a color profile, and an analysis of the stellar kinematics are given in Section 3. We discuss NGC 7025's pseudo-bulge formation and provide a brief summary in Section 5.

Throughout this paper, we assume a cosmological model with $H_{0}=70 \mathrm{~km} \mathrm{~s}^{-1} \mathrm{Mpc}^{-1}, \Omega_{\Lambda}=0.7$, and $\Omega_{\mathrm{m}}=0.3$. This yields a luminosity distance of $67.3 \mathrm{Mpc}$ and a scale of $316 \mathrm{pc} \operatorname{arcsec}^{-1}$ for NGC $7025\left(\mathrm{NED}^{7}\right)$.

\section{Data \\ 2.1. MEGARA Spectroscopy}

\subsubsection{MEGARA}

MEGARA $^{8}$ (Multi-Espectrógrafo en GTC de Alta Resolución para Astronomía) is a new optical IFU and multi-object spectrograph (MOS) installed on the $10.4 \mathrm{~m}$ Gran Telescopio CANARIAS (GTC) in La Palma (G. de Paz et al. 2018, in preparation). Both the MEGARA IFU and MOS have low, medium, and high spectral resolutions $R_{\text {spec }}$ of $\sim 6000,12,000$, and 20,000, respectively. The MEGARA IFU/MOS VPH gratings cover a wavelength range of $~ 3653-9686 \AA$.

The MEGARA IFU encompasses 567 contiguous hexagonal fibers, each with a long diagonal of $0 . " 62$, resulting in an $\sim 12.5 \times 11.3 \operatorname{arcsec}^{2}$ field of view in the shape of a rectangle. A total of eight static fiber bundles with 56 dedicated hexagonal fibers, located at the outermost parts of the field of view far from the IFU $\left(1 ! 5 \lesssim R \lesssim 2^{\prime}\right)$, deliver simultaneous sky observations.

\subsubsection{MEGARA Observations}

MEGARA IFU spectra of NGC 7025 were obtained in all VPH gratings as part of the instrument's commissioning run between 2017 June 24 and August 31. In this paper, we focus only on the high-resolution and low-resolution spectra of the galaxy obtained in MEGARA's VPH863-HR grating (henceforth referred to as HR-I) and VPH570-LR grating (henceforth, LR-V), respectively (Figures 1 and 2). The HR-I spectra have a wavelength range $\sim 8372-8882 \AA$, while the LR-V spectra cover $\sim 5144-6168 \AA$. This yields reciprocal linear dispersions of $0.13 \AA$ pixel $^{-} 1$ and $0.27 \AA$ pixel $^{-1}$ for the HR-I and LR-V spectra, respectively. For the HR-I spectra, the full width at half-maximum (FWHM) resolution at the central wavelength is $0.42 \AA \approx 15 \mathrm{~km} \mathrm{~s}^{-1}$, while for LR-V, FWHM $\sim 0.95 \AA \approx$ $50 \mathrm{~km} \mathrm{~s}^{-1}$ (Gil de Paz et al. 2016).

The LR-V and HR-I spectra of NGC 7025 were obtained on the nights of 2017 August 1 and 2, respectively. We obtained three exposures of NGC 7025 per VPH: $3 \times 900 \mathrm{~s}$ for both the HR-I and LR-V VPH gratings. The seeing conditions ranged between 0.7 and 1 ". 1 . To flux-calibrate the HR-I spectra, $3 \times 45$ s exposures of the flux standard star BD +174708 were obtained under seeing and airmass conditions similar to those of the science exposures. For the LR-V spectra, we obtained $3 \times 30 \mathrm{~s}$ exposures of the standard star BD+332642. We also obtained calibration data, including ThNe and ThAr arc lamps, halogen lamps, and twilight spectra.

\subsubsection{Data Reduction}

The raw IFU spectra of NGC 7025, "cleaned" of cosmic rays by interactively using the CLEANEST" (N. Cardiel et al. 2018,

\footnotetext{
https://ned.ipac.caltech.edu

8 https://guaix.fis.ucm.es/megara

9 http://cleanest.readthedocs.io/en/latest/
} 


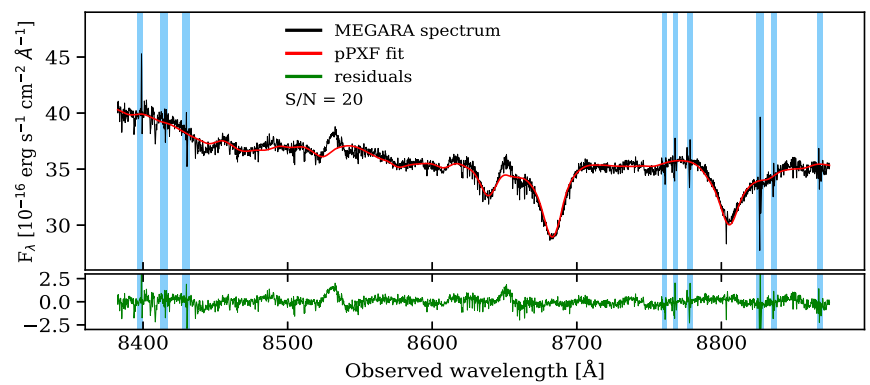

Figure 1. High-resolution MEGARA HR-I spectrum of NGC 7025, containing the $\mathrm{Ca}$ triplet absorption lines, extracted by co-adding all spaxels within the inner $1^{\prime \prime}$ radius (black curve). The red and green curves show the pPXF best fit to the spectrum and the associated residuals, respectively (see Section 3.3.1). The blue vertical stripes are the mask of sky emission regions and absorption features omitted from the pPXF fit.

in preparation) software package, were processed using the MEGARA data reduction pipeline ${ }^{10}$ (MDRP; S. Pascual 2018, in preparation). First, we masked the bad pixels in the IFU and subtracted a bias frame. The spectra were then processed using the MDRP tasks TRACE and MODELMAP by tracing the fibers across the flat halogen lamp frames. MDRP was used to perform the wavelength calibration of the HR-I and LR-V spectra using ThNe and ThAr arc lamp frames, respectively, to an accuracy of $\lesssim 0.01 \AA$ and $\lesssim 0.03 \AA$ rms values. MDRP corrects for spaxel-to-spaxel sensitivity and fiber-to-fiber transmission variations using our halogen and twilight flat frames, respectively. The spectra were flux-calibrated by comparing our observations of the flux standard star with the star's calibrated spectra provided by the CALSPEC calibration database. ${ }^{11}$ Finally, MDRP subtracts a median sky spectrum, determined using spectra from the 56 dedicated sky fibers, to generate fully reduced row-stacked spectra (RSS), where the rows pertain to the spectra of the individual $623(=567+56)$ science and sky fibers. The MDRP task CUBE transforms these RSS files into data cubes, but we use the RSS data for the stellar kinematic study in this paper. Figures 1 and 2 show the reduced central $\left(R \lesssim 1^{\prime \prime}\right)$ spectra of NGC 7025 .

\subsection{SDSS Imaging Data}

There are no high-resolution Hubble Space Telescope (HST) images available for NGC 7025. Therefore, 13 '.51 $\times 9$ '.83 biassubtracted and flat-fielded SDSS $g_{-}$and $i$-band images of NGC 7025 were retrieved from the SDSS Data Release 7 (DR7) database. ${ }^{12}$ These images contain a "soft bias" of $1000 \mathrm{DN}$, added to each pixel. In order to better correct for the galaxy's nuclear dust spiral, we used the $i$-band image for the detailed structural analysis (see Figures 3 and 4). We used the $g$ - and $i$-band data to extract the $g-i$ color profile of the galaxy (Figure 3).

\subsubsection{Data Reduction}

The SDSS images were reduced using standard IRAF tasks (Dullo et al. 2017, 2018, references therein). Subtracting the "soft bias," we determined the sky background levels as the average of the medians of the sky values from several $10 \times 10$ pixel boxes, far from the galaxy. An initial mask was generated

\footnotetext{
$\overline{10}$ http://megara-drp.readthedocs.io/en/latest/

$11 \mathrm{http}: / /$ www.stsci.edu/hst/observatory/crds/calspec.html

12 http://www.sdss.org
}

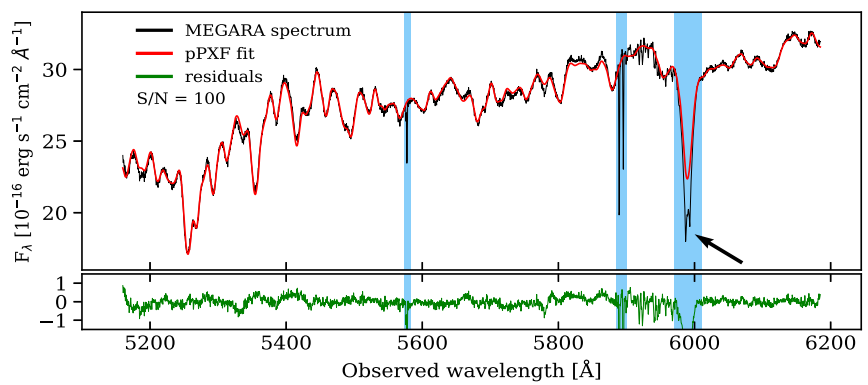

Figure 2. Similar to Figure 1 but now showing the MEGARA LR-V spectrum of NGC 7025 and the corresponding pPXF fit together with the residuals about this fit. The arrow indicates the resolved interstellar $\mathrm{Na} \mathrm{D}$ doublet. The blue vertical stripes show the mask of regions omitted from the pPXF fit.



Figure 3. Major-axis SDSS $i$-band surface brightness $\left(\mu_{i}\right)$, isophote shape parameter $\left(B_{4}\right)$, ellipticity $(\epsilon)$, position angle (P.A., measured in degrees from north to east), and color profiles of NGC 7025. Note that the $x$-axis is on a logarithmic scale. The typical errors associated with $B_{4}, \epsilon$, and P.A. are $20 \%-$ $30 \%, 4 \%$, and $5 \%$, respectively. The errors on $\mu_{i}$ and $g-i$ color are typically $0.015 \mathrm{mag} \operatorname{arcsec}^{-} 2$ and $0.02 \mathrm{mag}$. The dashed curves show the fourcomponent decomposition of the major-axis light profile, i.e., inner point source (green curve) + pseudo-bulge (red curve) + intermediate spiral-arm component (orange curve) + outer disk (blue curve). The solid curve (magenta) shows the complete fit with a small rms residual $\Delta$.

by running SEXTRACTOR (Bertin \& Arnouts 1996) and was then combined with a manual mask to avoid the dust spiral in the galaxy as well as the bright foreground stars, background galaxies, and chip defects in the images (Figures 4 and 5). We used this mask and fit the IRAF task ELLIPSE (Jedrzejewski 1987) to the sky-subtracted images following the steps outlined in Dullo et al. $(2017,2018)$. The results of the ELLIPSE fitting 

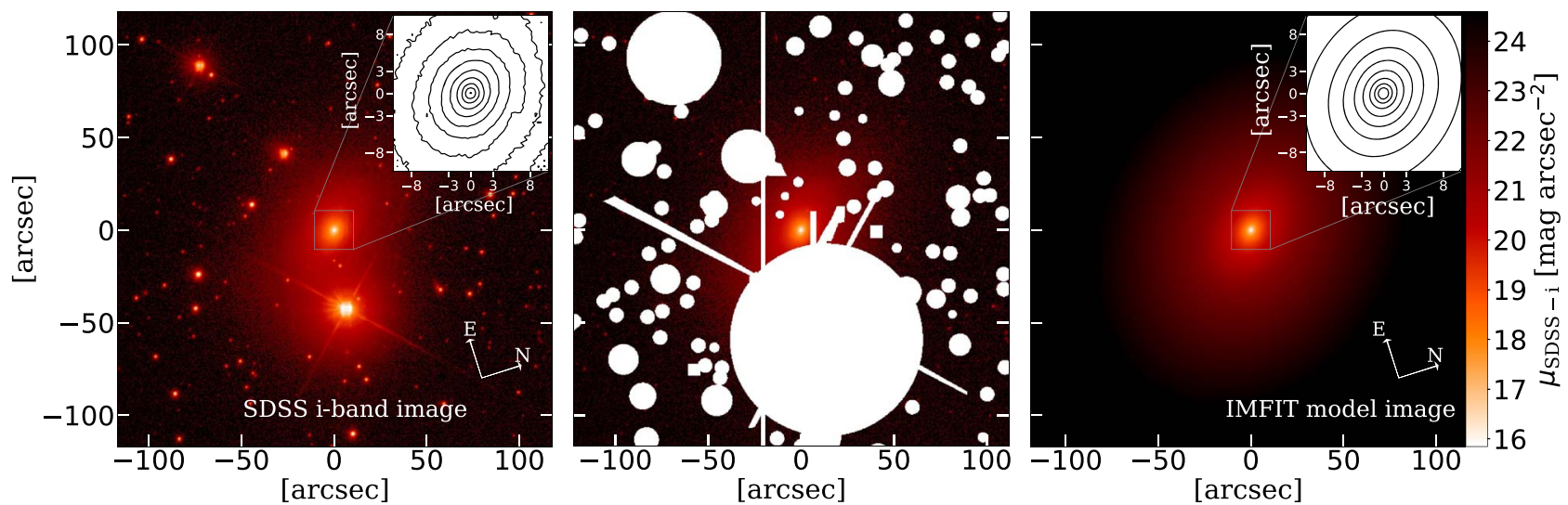

Figure 4. Left panel: SDSS $i$-band image of NGC 7025. Middle panel: masked regions (i.e., white areas). Right panel: IMFIT model image of NGC 7025. The insets show the surface brightness contours.


Figure 5. The residual images which are generated after subtracting the IRAF/BMODEL and IMFIT model images from the original SDSS image of NGC 7025 reveal dust spiral patterns and two tightly wound stellar spiral arms.

were fed into the IRAF task BMODEL to create a model image of the galaxy. This model image was subtracted from the science image to create an initial residual image and an improved mask (Figure 4, middle). We then reran the ELLIPSE fitting and BMODEL. Figure 3 shows the results of the ELLIPSE fitting, including the major-axis surface brightness, isophote shape parameter $\left(B_{4}\right)$, ellipticity, and position angle profiles of NGC 7025. Figure 5 (left) shows the final residual image. The surface brightnesses were calibrated using the zero-points, extinction coefficients, and airmass values given in the DR7 tsField files.

\section{Results}

\subsection{Photometric Structural Analysis}

Figure 3 shows our 1D four-component decomposition of the SDSS $i$-band surface brightness profile of NGC 7025. The fit, which contains a Gaussian $(n=0.5)$ nuclear component, a Sérsic bulge, a Sérsic intermediate-scale component, and an outer exponential $(n=1)$ disk, describes the light profile very well as revealed by the small $\mathrm{rms}$ residual value of $\sim 0.023 \mathrm{mag}$ $\operatorname{arcsec}^{-2}$ (Figure 3). This detailed multicomponent fit was performed following procedures similar to those in Dullo et al. (2017, 2018). For each component, the model profile was convolved with a Gaussian point-spread function (PSF) with an FWHM $\sim 0$ ".98. The FWHM of the PSF, which we measured using several stars in the SDSS $i$-band image, agrees with that given in the SDSS DR7 tsField file.

We additionally performed a 2D four-component decomposition of the SDSS $i$-band image using IMFIT $^{13}$ (Erwin 2015). We used the result from the 1D fitting as an input for the 2D fitting. To convolve the model images, we used a Moffat PSF image generated using the IMFIT task MAKEIMAGE. Figure 4 (right) shows the best-fitting 2D Gaussian nuclear component + Sérsic bulge + Sérsic intermediate-scale component + outer exponential disk model image that describes the 2D light distribution of the galaxy. IMFIT subtracted this model image from the galaxy image to produce the residual image (Figure 5, right). Tables 1 and 2 list the best-fitting model parameters from the $1 \mathrm{D}$ and $2 \mathrm{D}$ decompositions.

Both the ELLIPSE and IMFIT residual images reveal a dust spiral pattern which extends from the galaxy center out to $R \sim 24^{\prime \prime}$, indicative of recent star formation activity in the galaxy. Also apparent in these residual images are two tightly wound stellar spiral arms over the regions where the intermediate-scale component dominates the bulge light. This Sérsic intermediate-scale (spiral-arm) component with Sérsic index $n \sim 0.22$, dominated by the outer disk at all radii, is also evidenced by the disky isophotes (i.e., the ELLIPSE $B_{4}>0$ and IMFIT $\left.c_{0}<0\right)$ and high ellipticity $(\epsilon \sim 0.35)$ at 22 "' $5 \lesssim R \lesssim 32^{\prime \prime}$. As can be seen in Figure 3, the ellipticities

\footnotetext{
${ }^{13}$ http://www.mpe.mpg.de/ erwin/code/imfit/
} 
Table 1

Structural Parameters

\begin{tabular}{|c|c|c|c|c|c|c|c|c|c|c|c|c|c|c|}
\hline \multicolumn{15}{|c|}{ 1D Fit (Major Axis) } \\
\hline $\begin{array}{l}\mu_{\mathrm{e}, \text { bul }} \\
\text { (1) }\end{array}$ & $\begin{array}{c}R_{\mathrm{e}, \text { bul }} \\
(\operatorname{arcsec}) \\
(2)\end{array}$ & $\begin{array}{c}R_{\mathrm{e}, \mathrm{bul}} \\
(\mathrm{kpc}) \\
(3)\end{array}$ & $\begin{array}{l}n_{\text {bul }} \\
(4)\end{array}$ & $\begin{array}{c}\mu_{\mathrm{e}, \text { int }} \\
\text { (5) }\end{array}$ & $\begin{array}{c}R_{\mathrm{e}, \text { int }} \\
(\operatorname{arcsec}) \\
\quad(6)\end{array}$ & $\begin{array}{l}n_{\text {,int }} \\
(7)\end{array}$ & $\begin{array}{c}\mu_{0, \text { disk }} \\
(8)\end{array}$ & $\begin{array}{c}h \\
(\operatorname{arcsec}) \\
(9)\end{array}$ & $\begin{array}{c}m_{\mathrm{pt}} \\
(\mathrm{mag}) \\
(10)\end{array}$ & $\begin{array}{c}M_{i, \text { bul }} \\
(\mathrm{mag}) \\
(11)\end{array}$ & $\begin{array}{l}B / T \\
(12)\end{array}$ & $\begin{array}{l}B / D \\
(13)\end{array}$ & $\begin{array}{c}M / L_{i, \text { bul }} \\
(14)\end{array}$ & $\begin{array}{c}\log \left(M_{*, \text { bul }} / M_{\odot}\right) \\
(15)\end{array}$ \\
\hline 18.77 & 5.23 & 1.65 & 1.80 & 22.04 & 20.15 & 0.21 & 20.55 & 28.89 & 16.95 & -22.53 & 0.30 & 0.48 & 0.63 & 10.62 \\
\hline \multicolumn{15}{|c|}{ 2D Fit (IMFIT) } \\
\hline & $20 \%$ & & $15 \%$ & & $25 \%$ & $20 \%$ & & $15 \%$ & & & $15 \%$ & $15 \%$ & $15 \%$ & $40 \%$ \\
\hline
\end{tabular}

Notes. 1D and 2D structural parameters from the Gaussian point source + Sérsic pseudo-bulge + Sérsic intermediate component + exponential outer disk model fit to the SDSS $i$-band major-axis light profile and to the SDSS $i$-band image of NGC 7025, respectively (Figures 3-5). Col. 1-7: Sérsic model parameters for the pseudobulge and intermediate-scale component. Col. 8-9: best-fitting parameters for the outer exponential disk. Col. 10: apparent magnitude of the point source. Col. 11: absolute magnitude of the pseudo-bulge calculated using Col. 1-3 and corrected for galactic extinction, surface brightness dimming, and internal dust attenuation. Col. 12-13: bulge-to-total and bulge-to-disk flux ratios. The above dust and surface brightness dimming corrections were not applied to these flux ratios. Col. 14-15: $i$-band mass-to-light ratio $\left(M / L_{i, \text { bul }}\right)$ and stellar mass of the pseudo-bulge $\left(M_{*, \text { bul }}\right) . \mu_{\mathrm{e}}$ and $\mu_{0}$ are in mag $\operatorname{arcsec}^{-2}$. The last row shows the $1 \sigma$ uncertainties on the fit parameters, flux ratios, and stellar masses—see the text for details. The uncertainties associated with $m_{\mathrm{pt}}$ and $M_{\mathrm{i}, \mathrm{bul}}$ are 0.95 mag and 0.2 mag, respectively. The uncertainty on $\mu_{\mathrm{e}}$ and $\mu_{0}$ is $\sim 0.025 \mathrm{mag} \operatorname{arcsec}^{-2}$.

Table 2

Structural Parameters

\begin{tabular}{lccccccc}
\hline \hline & \multicolumn{7}{c}{$\begin{array}{c}\text { 2D Fit } \\
\text { (IMFIT) }\end{array}$} \\
\hline $\begin{array}{l}\text { P.A.bul } \\
\left({ }^{\circ}\right)\end{array}$ & $\epsilon_{\text {bul }}$ & $c_{0, \text { bul }}$ & $\begin{array}{c}\text { P.A.int } \\
\left({ }^{\circ}\right)\end{array}$ & $\epsilon_{\text {int }}$ & $c_{0, \text { int }}$ & $\begin{array}{c}\text { P.A.disk } \\
\left({ }^{\circ}\right)\end{array}$ & $\epsilon_{\text {disk }}$ \\
$(1)$ & $(2)$ & $(3)$ & $(4)$ & $(5)$ & $(6)$ & $(7)$ & $(8)$ \\
\hline 46.78 & 0.21 & -0.05 & 40.01 & 0.37 & -0.01 & 42.41 & 0.25 \\
\hline $10 \%$ & $10 \%$ & $10 \%$ & $10 \%$ & $10 \%$ & $10 \%$ & $10 \%$ & $20 \%$ \\
\hline
\end{tabular}

Notes. Similar to Table 1 but showing the remaining structural parameters from the 2D decomposition with IMFIT. The IMFIT isophote shape parameter $c_{0}$ as given by Erwin (2015, their Equation 25) is positive/negative for boxy/disky isophotes, not to be confused with the IRAF/ELLIPSE $B_{4}$.

and orientations of the (spiral-arm) component and outer disk from the ELLIPSE isophote fitting are, unsurprisingly, similar.

Given that we fit a Sérsic model to the intermediate-scale spiral-arm component, the flux and Sérsic index of the bulge as well as the flux of the outer disk may be biased to lower values. Concerned about this, we went further and performed a $2 \mathrm{D}$ four-component decomposition of the galaxy image using GALFIT (Peng et al. 2010; see Table 3 and Figure 6), fitting the GALFIT logarithmic spiral (log-tanh) model image (Figure 6, left; see Peng et al. 2010, their Equation 29) to the spiral-arm component. The GALFIT residual image in Figure 6 (right) shows some stellar spiral-arm structures that we missed in our modeling. However, we avoided performing a more sophisticated fit, due to strong degeneracies between the fit parameters. Our GALFIT and IMFIT decompositions agree very well except for the spiral-arm component model. The GALFIT best-fitting parameters for the point source and outer disk are similar to those from IMFIT; thus we do not show them in Table 3 . The best-fitting parameters of the bulge (e.g., $n, R_{\mathrm{e}}$, and $M_{i}$ ) from GALFIT are in good agreement with those from IMFIT (see Tables $1-3)$. For example, $n_{\text {bul,GALFIT }}(\sim 1.92 \pm 0.30)$ agrees well within the uncertainties with $n_{\text {bul,IMFIT }}(\sim 1.52 \pm 0.23)$.

The agreement between our 1D and 2D decompositions is very good (Figure 5, Tables 1 and 2), although the apparent magnitude of the innermost nuclear component, obtained using the best-fitting 1D (major-axis) structural parameters and the pertinent ellipticity $(\sim 17.0 \mathrm{mag})$, differs somewhat from that of the $2 \mathrm{D}$ fit $(\sim 16.0 \mathrm{mag})$. Since this faint Gaussian nuclear component (which accounts for $0.5 \%-1.1 \%$ of the total galaxy light) is round $(\epsilon \sim 0.12)$, we identify it as a point source (e.g., Dullo \& Graham 2015). However, the disky isophotes inside 0 " 8 may suggest the presence of a nuclear disk (instead of a point source) that is poorly constrained due to the nuclear dust spiral and PSF of the SDSS data.

To determine the uncertainties on the fit parameters and associated quantities (magnitudes, flux ratios, and stellar masses), we ran a series of Monte Carlo simulations. We took into account the rms residual scatter of the fit (Figures 3, 4, and 6); possible contamination of the surface brightness profile, due to the bright foreground star and dust spiral; and errors from incorrect sky subtraction to create 200 realizations of the galaxy's light profile. Each of these realizations was decomposed into four components in a manner similar to the aforementioned modeling of the actual light profile of the galaxy. Tables 1 and 2 show the $1 \sigma$ uncertainties calculated using the best-fitting parameters from the 200 light profile decompositions.

\subsection{Bulge Identification: Pseudo versus Classical}

This work focuses on NGC 7015's bulge. Importantly, we want to determine whether the bulge is a "classical bulge" formed through violent merger processes or a "pseudo-bulge" built out of the disk material via secular evolution or formed by other mechanisms. Here, we do so by considering the Sérsic index, ellipticity, position angle (P.A.), $B_{4}$, and bulge-to-total flux ratio $(B / T)$ of the galaxy.

Having an ellipticity $\epsilon_{\text {bul }} \sim 0.19_{-0.03}^{+0.06}$, the bulge with a low Sérsic index $n \sim 1.5-1.9$ and a major-axis half-light radius of $R_{\mathrm{e}} \sim 5$ !' $2 \approx 1.7 \mathrm{kpc}$ dominates the galaxy light in the inner regions ( $R \lesssim 15^{\prime \prime}$; Figures 3 and 4 and Tables 1 and 2$)$. We note that most pseudo-bulges have a Sérsic index $n<2$, while most classical bulges have $n>2$ (e.g., Kormendy \& Kennicutt 2004; Kormendy 2016). The outer disk with $\epsilon_{\text {disk }} \sim 0.32_{-0.07}^{+0.04}$ dominates at $R>15^{\prime \prime}$. Secularly built bulges tend to have 

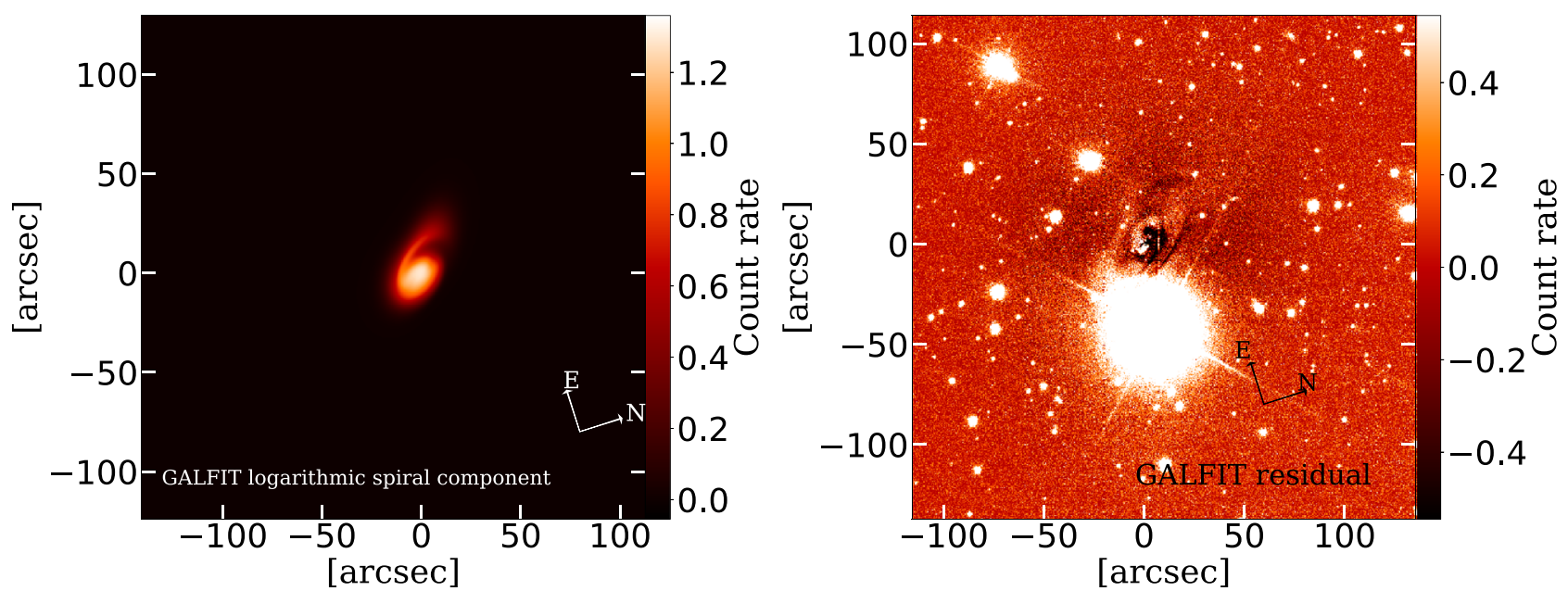


model image of NGC 7025, which contains the spiral-arm component (left panel), from the original SDSS image of the galaxy.

ellipticities and position angles similar to those of their associated outer disks (e.g., Kormendy \& Kennicutt 2004; Fisher \& Drory 2008). However, pseudo-bulges, akin to classical bulges, can be rounder than their disks (e.g., Fisher \& Drory 2008, their Section 4; Erwin et al. 2015; Dullo et al. 2016). Fisher \& Drory (2008) noted that the formation of pseudo-bulges that are flatter than their outer disks can be due to bar bucklings and/or unstable disks which move stars above the plane of the disks (e.g., Pfenniger \& Norman 1990; Martinez-Valpuesta et al. 2006). For NGC 7025, $\epsilon_{\text {bul }} / \epsilon_{\text {disk }} \sim 0.59_{-0.16}^{+0.20}$. The ratio of the average ellipticity of the bulge to that of the disk for $30 \%$ of the 53 pseudo-bulges in Fisher \& Drory (2008, their Figure 11 and Table 3 ) is $\left\langle\epsilon_{\text {bul }}\right\rangle /\left\langle\epsilon_{\text {disk }}\right\rangle \lesssim 0.60$. For about half of their pseudo-bulges, the value of $\left\langle\epsilon_{\text {bul }}\right\rangle /\left\langle\epsilon_{\text {disk }}\right\rangle$ or $\left\langle\epsilon_{\text {disk }}\right\rangle /\left\langle\epsilon_{\text {bul }}\right\rangle$ is $\lesssim 0.70$, and for $10 \%$ of their pseudo-bulges, $\left\langle\epsilon_{\text {bul }}\right\rangle /\left\langle\epsilon_{\text {disk }}\right\rangle \sim 0.60$, similar to that of NGC 7025. Omitting the most PSF-affected region, the position angle of the bulge of NGC 7025 is only modestly (i.e., $5^{\circ}-10^{\circ}$ ) twisted from that of the outer disk (Figure 3; see also Figure 4 and Table 2). Pseudo-bulges displaying such degrees of alignment with their outer disks are presented in Kormendy \& Kennicutt (2004, their Figures 10 and 12), Erwin et al. (2015, their Figures 3 and 5), and Dullo et al. (2016, their Figure 4), among others. Importantly, the outer part of NGC 7025's bulge has the same position angle as its disk.

The quantity $B_{4}$ in the output of IRAF/ELLIPSE quantifies the deviations of isophotes from pure ellipses: $B_{4}$ is negative/ positive for boxy/disky isophotes (Jedrzejewski 1987). Outside the PSF-affected region, the bulge of NGC 7025 is disky inside $R \sim 5^{\prime \prime}$, turning into boxy at $6^{\prime \prime} \lesssim R<10^{\prime \prime}$ before becoming disky again over $10^{\prime \prime} \lesssim R \lesssim 15^{\prime \prime}$. The $B_{4}$ values of the bulge's disky isophotes are typically $\sim 0.010$, with $20 \%-30 \%$ associated uncertainties, in fair agreement with those of the disky isophotes from a large portion of the galaxy's disk-dominated region $\left(B_{4} \sim 0.007-0.015\right.$; see Figure 3$)$. Note that the values of $\left|B_{4}\right|$ are typically $<0.02$ for elliptical, lenticular, and earlytype spiral galaxies (e.g., Graham et al. 2012, their Figure 4), but disky/boxy isophotes of galaxies with bars, dust, and/or prominent spiral structures can have $\left|B_{4}\right|$ values $>0.02$. Although disky isophotes in disk galaxies are primarily due to disks, the presence of bars, rings, and strong spiral arms can also result in disky isophotes. However, the latter are often accompanied by strong isophotal twists and local extrema in ellipticities (e.g., see Dullo et al. 2016).

From the integration of the $1 \mathrm{D}$ and $2 \mathrm{D}$ model components, accounting for the ellipticity of the 1D components, we measured the galaxy's bulge-to-total and bulge-to-disk flux ratios to be $B / T \sim 0.28-0.30$ and $B / D \sim 0.48$, respectively (Table 1). This is in excellent agreement with Erwin et al. (2015), who found a mean $B / T \sim 0.33$ for his sample of pseudo-bulges (see also Fisher \& Drory 2008, their Figure 11). The 1D decomposition (Figure 3) gives a dust-corrected SDSS $i$-band absolute magnitude for the bulge $M_{i \text {,bul }} \sim-22.53 \mathrm{mag}$, while our IMFIT and GALFIT 2D decompositions yield $M_{i, \text { bul }} \sim-22.59 \mathrm{mag}$ and $\sim-22.55 \mathrm{mag}$, respectively. We corrected the observed absolute bulge magnitudes for inclination and internal dust extinction (Driver et al. 2008, their Table 1 and Equations 1 and 2), galactic extinction, and surface brightness dimming. These $i$-band absolute magnitudes were converted into solar luminosities using an absolute magnitude for the Sun of $M_{i, \odot}=4.53 \mathrm{AB}$ mag. ${ }^{14}$ To convert the

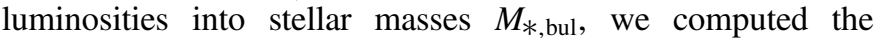
bulge's mass-to-light ratio $\left(M / L_{i, \text { bul }}\right)$ using its $g-i$ color of 1.40 mag (Figure 3 ) and the color $-(M / L)$ relation by Into $\&$ Portinari (2013, their Table 3 ). This yielded $M / L_{i \text {,bul }} \sim 0.63$; thus $M_{* \text {, bul }} \sim(4.21-4.47) \times 10^{10} M_{\odot}$.

In summary, the ellipticity, position angle, $n, B / T$, and predominantly disky isophotes of NGC 7025's bulge together with the presence of dust and stellar spiral structures in the galaxy (Figure 5) strongly suggest a modestly flattened pseudobulge. Indeed, Kormendy (2016) noted that classical bulges cannot have spiral structures. Building on our structural analysis, in Section 3.3 we present the stellar kinematics of the bulge.

\subsubsection{Comparison with Past Decomposition}

Recently, Neumann et al. (2017), Rizzo et al. (2018), and Gilhuly \& Courteau (2018) fit a 2D Sérsic bulge + exponential disk model to the SDSS images of NGC 7025 without accounting for the intermediate (spiral-arm) component. As warned by Dullo et al. (2016, 2017, 2018; see also Dullo \& Graham 2012, 2013, 2014; Graham et al. 2016), neglecting to

\footnotetext{
14 http://mips.as.arizona.edu/ cnaw/sun.html
} 
Table 3

Structural Parameters

\begin{tabular}{|c|c|c|c|c|c|c|c|c|}
\hline & & & & 2D Fit (GALFIT) & & & & \\
\hline Component & $\begin{array}{l}\text { Sérsic } \\
\log \\
\text { Fourier } \\
\text { (2) }\end{array}$ & $\begin{array}{c}m_{i}(\mathrm{mag}) \\
R_{\text {in }}\left({ }^{\prime \prime}\right) \\
\text { mode: amplitude } \\
\text { (3) }\end{array}$ & $\begin{array}{c}n \\
R_{\text {out }}\left({ }^{\prime \prime}\right) \\
\text { phase }\left(^{\circ}\right) \\
(4)\end{array}$ & $\begin{array}{c}R_{\mathrm{e}}\left({ }^{\prime \prime}\right) \\
\theta_{\text {rot }}\left({ }^{\circ}\right) \\
\text { mode: amplitude } \\
(5)\end{array}$ & $\begin{array}{c}\text { P.A. }\left({ }^{\circ}\right) \\
R_{\mathrm{ws}}\left({ }^{(\prime)}\right) \\
\text { phase }\left({ }^{\circ}\right) \\
(6)\end{array}$ & $\begin{array}{c}\epsilon \\
\theta_{\text {incl }}\left({ }^{\circ}\right) \\
(7)\end{array}$ & $\begin{array}{c}c_{0} \\
\theta_{\text {sky }}\left({ }^{\circ}\right) \\
(8)\end{array}$ & $M_{i}(\mathrm{mag})$ \\
\hline $\begin{array}{l}\text { Bulge } \\
\text { Intermediate spiral-arm } \\
\text { component }\end{array}$ & $\begin{array}{l}\text { Sérsic } \\
\text { Sérsic } \\
\log \\
\text { Fourier }\end{array}$ & $\begin{array}{l}12.40 \\
14.26 \\
18.61 \\
1: 0.40\end{array}$ & $\begin{array}{l}1.92 \\
0.45 \\
5.54 \\
0.22\end{array}$ & $\begin{array}{c}5.21 \\
16.22 \\
80.8 \\
2: 0.36\end{array}$ & $\begin{array}{c}45.49 \\
47.79 \\
2.61 \\
9.15\end{array}$ & $\begin{array}{c}0.20 \\
0.42 \\
52.69 \\
\ldots\end{array}$ & $\begin{array}{c}-0.01 \\
\ldots \\
54.82 \\
\ldots\end{array}$ & $\begin{array}{c}-22.55 \\
\ldots \\
\ldots \\
\ldots\end{array}$ \\
\hline
\end{tabular}

Notes. Similar to Tables 1 and 2 but showing here the bulge and spiral-arm structural parameters from the 2D decomposition of the SDSS $i$-band image of NGC 7025 with GALFIT (Peng et al. 2010, their Equation 29). The GALFIT best-fitting parameters for the point source and outer disk are similar to those from IMFIT. We fit the GALFIT logarithmic spiral (log-tanh) function to the intermediate spiral-arm component—see the text for details. $m_{i \text {,bul }}$ is the apparent magnitude of the bulge returned by GALFIT. $M_{i \text {,bul }}$ is the absolute magnitude of the bulge calculated using $m_{i, \text { bul }}$ and corrected for galactic extinction, surface brightness dimming, and internal dust attenuation. $R_{\text {in }}$ and $R_{\text {out }}$ are the inner and outer radii of the spiral with cumulative rotation of $\theta_{\text {rot }}$ and a winding-scale radius of $R_{\mathrm{ws}} . \theta_{\mathrm{incl}}$ and $\theta_{\mathrm{sky}}$ are the spiral's sky inclination and position angles, respectively. Errors on the Sérsic fit parameters are as in Tables 1 and 2.
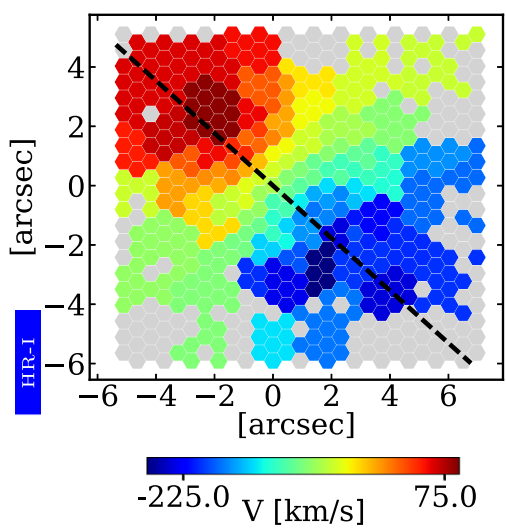

(a)

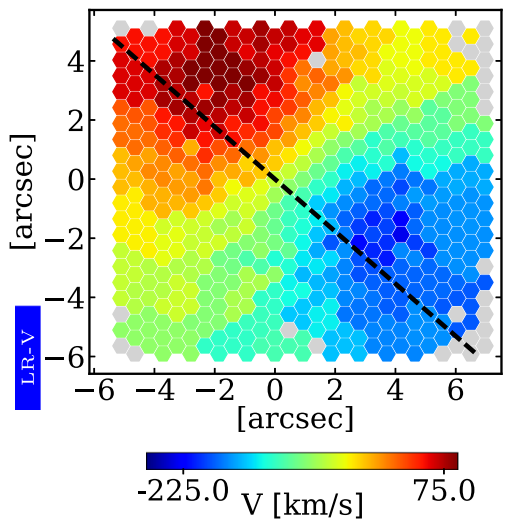

(e)
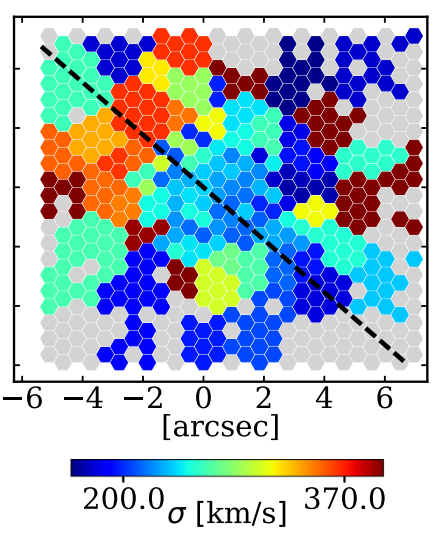

(b)



(f)

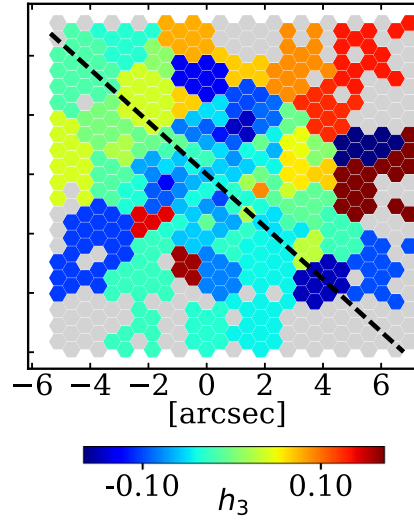

(c)



(g)



$(\mathrm{d})$

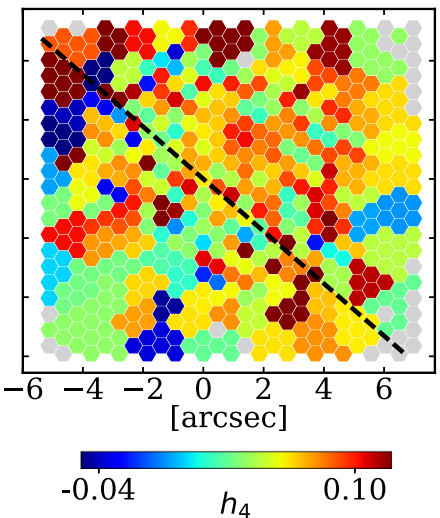

$(\mathrm{h})$

Figure 7. MEGARA stellar kinematic maps of NGC 7025 extracted from the HR-I spectra (top row) and LR-V spectra (bottom row). These maps extend to roughly the semimajor-axis half-light radius of the pseudo-bulge $\left(R_{\mathrm{e}, \text { maj }} \sim 55^{\prime \prime} 23\right)$. We excluded fibers with $\mathrm{S} / \mathrm{N}<3$, shown in light gray, and co-added the HR-I data into spatial bins with S/N > 7 using the Voronoi binning algorithm (Cappellari \& Copin 2003). The LR-V data were similarly co-added into spatial bins withS/N > 15. The panels show the velocity $V$ determined with respect to NGC 7025's systemic velocity ((a) and (e)), the velocity dispersion $\sigma$ ((b) and (f)), and the higher-order Gauss-Hermite coefficients $h_{3}((\mathrm{c})$ and $(\mathrm{g}))$ and $h_{4}((\mathrm{~d})$ and $(\mathrm{h}))$. The dashed lines show the photometric major axis of the pseudo-bulge, which was determined excluding the PSF-affected region (see Figure 3). North is up and east is to the left.

fit intermediate-scale components (including bars, disks, and spiral arms) as a separate component can systematically bias the Sérsic index and flux of the inner bulge toward higher values. Indeed, Neumann et al. (2017) argued that their Sérsic index for the bulge of NGC $7025(n \sim 2.7)$, the bulge's position in the Kormendy relation, and the galaxy's concentration index and velocity dispersion gradient all favor a merger-built "classical" bulge with $B / T \sim 0.44$. Similarly, due to the flux of the intermediate (spiral-arm) component which was attributed to the bulge, the bulge-disk decomposition by Gilhuly \& Courteau (2018) yielded a high Sérsic index for the bulge $(n \sim 5.1)$, indicating a slow-rotating classical 
bulge. Rizzo et al. (2018) classify NGC 7025 as an early-type S0a. Their modeling of the bulge light distribution for this galaxy yielded $n \sim 2.4$ and $B / T \sim 0.37$; both these figures are larger than ours (see Tables 1 and 3 ).

\subsection{Spectroscopy}

\subsubsection{Stellar Kinematics}

The MEGARA HR-I and LR-V spectra for NGC 7025 have a high signal-to-noise ratio per spaxel $(\mathrm{S} / \mathrm{N})$ of $12-100$ at the central regions, compared to the low $\mathrm{S} / \mathrm{N}$ of $\sim 1-5$ at the outer parts of the IFU. We excluded all fibers with $\mathrm{S} / \mathrm{N}<3$, which are shown in light gray in Figure 7, and spatially binned the data using the 2D Voronoi binning technique by Cappellari \& Copin (2003). For the HR-I spectra, we found that forcing each bin's minimum $\mathrm{S} / \mathrm{N}$ threshold to 7 results in a reliable measurement of the stellar rotation and stellar velocity dispersion for the pseudo-bulge of NGC 7025, with optimum spatial resolution over $R \lesssim 7^{\prime \prime}$. For the LR-V spectra, with $\mathrm{S} / \mathrm{N}$ higher than that of the HR-I data, a minimum $\mathrm{S} / \mathrm{N}$ threshold of 15 allows a robust stellar kinematic measurement for the pseudo-bulge.

We fit the binned MEGARA HR-I and LR-V spectra with the E-MILES and MILES stellar templates, ${ }^{15}$ respectively (Vazdekis et al. 2016), to determine the stellar kinematics (the stellar rotation $V$, stellar velocity dispersion $\sigma$, skewness $h_{3}$, and kurtosis $h_{4}$ ) of the galaxy using the penalized pixel-fitting code (pPXF) by Cappellari \& Emsellem (2004; see also Cappellari 2017; Figures 1, 2, and 7). These template spectra span a range of ages $(0.063 \mathrm{Gyr}$ to $17.78 \mathrm{Gyr})$ and metallicities $(-2.32<[\mathrm{M} / \mathrm{H}]<+0.22)$. We adopted a Salpeter initial mass function (Salpeter 1955) for this massive spiral galaxy as suggested by Dutton et al. (2013). The E-MILES/MILES templates that we used have a spectral resolution of FWHM $=2.50 \AA$ and velocity dispersions $\sigma \sim 40 \mathrm{~km} \mathrm{~s}^{-} 1$ and $60 \mathrm{~km} \mathrm{~s}^{-1}$ at the central wavelengths of the MEGARA HR-I and LR-V spectra, respectively (Vazdekis et al. 2016, their Figure 8). These can be compared to the MEGARA spectral resolutions of FWHM $\sim 0.42 \AA \approx 15 \mathrm{~km} \mathrm{~s}^{-} 1$ and FWHM $\sim 0.95 \AA \approx 50 \mathrm{~km} \mathrm{~s}^{-1}$ for the HR-I and LR-V spectra, respectively (see Section 2.1.2). Given that NGC 7025's bulge has broad stellar absorption features (i.e., $\sigma \gtrsim 155 \mathrm{~km} \mathrm{~s}^{-1}$ ), it was not necessary to convolve the highresolution MEGARA spectra to degrade them to the resolution of the E-MILES/MILES template spectra (Figures 1, 2, and 7).

The high-resolution MEGARA stellar velocity $(V)$ maps of NGC 7025, which extend to roughly the major-axis half-light radius of the pseudo-bulge $\left(R_{\mathrm{e} \text {,maj }} \sim 5\right.$ "' 23$)$, reveal a significant rotation around the photometric minor axis of the galaxy, rising up to $|V| \sim 200 \mathrm{~km} \mathrm{~s}^{-1}$ (Figures 7(a) and (e)). The pseudobulge has a stellar velocity dispersion of $\sigma \sim 230 \mathrm{~km} \mathrm{~s}^{-1}$ near the center (i.e., at $R \lesssim 0.5 R_{\mathrm{e}}$; Figures 7(b) and (f)). At larger radii $\left(0.5 R_{\mathrm{e}} \lesssim R \lesssim R_{\mathrm{e}}\right)$, the $\sigma$ map extracted using the HR-I data shows that large parts of the pseudo-bulge display $\sigma \sim 270-350 \quad \mathrm{~km} \mathrm{~s}^{-1}$, but there are regions with $\sigma \sim 155-220 \mathrm{~km} \mathrm{~s}^{-1}$ (Figure 7(b)). In contrast, the $\sigma$ map based on the LR-V spectra shows that the pseudo-bulge typically has $\sigma \sim 155-220 \mathrm{~km} \mathrm{~s}^{-1}$ at $0.5 R_{\mathrm{e}} \lesssim R \lesssim R_{\mathrm{e}}$. This discrepancy is primarily due to the contrasting $\mathrm{S} / \mathrm{N}$ of the HR-I

\footnotetext{
15 http://miles.iac.es
}

and LR-V spectra, i.e., $(\mathrm{S} / \mathrm{N})_{\text {HR-I }} \sim 10-35$ versus $(\mathrm{S} /$ $\mathrm{N})_{\mathrm{LR}-\mathrm{V}} \sim 20-60$. The $\sigma$ maps are less reliably constrained for the HR-I data with modest $\mathrm{S} / \mathrm{N} \sim 10$. It is also in part because the HR-I spectra are weakly sensitive to localized dust absorption in the central regions of the galaxy, compared to the LR-V spectra. These detailed MEGARA $V$ and $\sigma$ maps together with the Falcón-Barroso et al. (2017, their Figures A10 and A27) low-resolution CALIFA $V$ and $\sigma$ maps, with larger radial extent reaching $R \sim 30^{\prime \prime}$, reveal kinematic regularity in the galaxy - that is, an overall outwardly increasing (declining) rotation (stellar velocity dispersion). In agreement with our decomposition, these large field-of-view kinematic maps by Falcón-Barroso et al. (2017) show that NGC 7025 exhibits a high degree of rotation $\left(|V|>150 \mathrm{~km} \mathrm{~s}^{-1}\right)$ at $R \gtrsim 25^{\prime \prime}$, i.e., at radii where the outer disk dominates the pseudo-bulge.

Because the HR-I spectra have somewhat low $\mathrm{S} / \mathrm{N}$ at $R \gtrsim 0.5 R_{\mathrm{e}}$, the corresponding $h 3$ and $h_{4}$ values are poorly constrained (Figures 7(c) and (d)). For the LR-V spectra, the $h_{3}$ map is anticorrelated with the $V$ map, as expected for disky rotating systems (Figure 7; see also Bender et al. 1994; González-García et al. 2006; Krajnović et al. 2008; van de Sande et al. 2017). The pseudo-bulge typically has $h_{4}>0$ at $R \lesssim R_{\mathrm{e}}$ (Figure 7(h)).

$$
\text { 3.3.2. } V / \sigma
$$

The disky pseudo-bulge in NGC 7025 is evident from the decompositions and isophotal analysis in Section 3.1. The correspondence between the central structure of early-type galaxies and their kinematics results in the disky/boxy, "fast rotator" "slow rotator" dichotomy (e.g., Bender et al. 1989; Franx et al. 1991; Faber et al. 1997). The former are thought to structurally and kinematically resemble the rotating bulges in spiral galaxies. The ratio of stellar rotation to velocity dispersion $(V / \sigma)$ together with ellipticity $\epsilon$ has been used to determine whether a system is supported by rotation or by anisotropic velocity dispersion (e.g., Illingworth 1977; Kormendy 1982; Davies et al. 1983). We followed the recent approach by Fabricius et al. (2012) and Erwin et al. (2015): first correct $V$ for inclination $(i)$ as $V_{\text {corr }}=V / \sin (i)$ and then determine $V_{\text {corr }} / \sigma$ for NGC 7025 with $i \sim 53^{\circ} .3$ (HyperLeda, ${ }^{16}$ Paturel et al. 2003). We adopted a kinematic classification where rotation-supported systems have regions with $V_{\text {corr }} / \sigma \geqslant 1$, while for pressure-supported systems $V_{\text {corr }} / \sigma<1$ (e.g., Fabricius et al. 2012; Erwin et al. 2015). Figure 8 shows the HR-I and LR-V $V_{\text {corr }} / \sigma$ maps of NGC 7025 's pseudo-bulge. These maps show that $\left|V_{\text {corr }} / \sigma\right|$ climbs to $\sim 1.5$, revealing that the pseudo-bulge is mainly supported by rotation.

\subsubsection{Specific Angular Momentum $\lambda_{R}$}

Using the SAURON IFU data for a sample of 48 early-type galaxies, Emsellem et al. (2007) suggested the parameter $\lambda_{R}$ for discriminating between slow and fast rotators in their sample. This (spin) parameter $\lambda_{R}$, a proxy for the stellar angular momentum per unit mass, is defined as

$$
\lambda_{R_{\max }}=\frac{\sum_{i=1}^{N_{\max }} F_{i} R_{i}\left|V_{i}\right|}{\sum_{i=1}^{N_{\max }} F_{i} R_{i} \sqrt{V_{i}^{2}+\sigma_{i}^{2}}},
$$

\footnotetext{
${ }^{16}$ http://leda.univ-lyon1.fr/search.html
} 


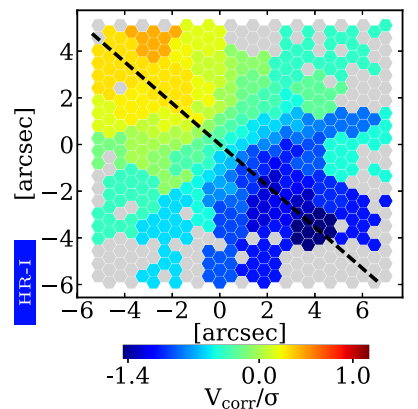

(a)



(b)
Figure 8. Similar to Figure 7 but here showing the ratios of the inclinationcorrected stellar rotation velocity $\left(V_{\text {corr }}\right)$ to the velocity dispersion $(\sigma)$ for the disky pseudo-bulge in NGC 7025. We corrected the stellar velocity for inclination $\left(i \sim 53.3^{\circ}\right)$ as $V_{\text {corr }}=V / \sin (i) .\left|V_{\text {corr }} / \sigma\right|$ rose to $\sim 1.5$, indicating that the pseudo-bulge is supported by rotation.

where $F_{i}, R_{i}, V_{i}$, and $\sigma_{i}$ are the flux, circular radius, velocity, and velocity dispersion of the $i$ th spatial bin, respectively. Having measured the half-light spin parameter $\lambda_{\mathrm{e}}=\lambda\left(R_{\mathrm{e}}=\right.$ 4.' 68) and average ellipticity of the galaxy within $R_{\mathrm{e}}\left(\epsilon_{\mathrm{e}}\right)$, in Figure 9 (left) we place NGC 7025 in the $\lambda_{\mathrm{e}}-\epsilon_{\mathrm{e}}$ diagram and compare it with the ATLAS ${ }^{3 \mathrm{D}}$ sample of 260 early-type galaxies (Emsellem et al. 2011). Fast rotators are defined by Emsellem et al. (2011) as those galaxies with $\lambda_{\mathrm{e}}>0.31 \times \sqrt{\epsilon}_{\mathrm{e}}$ and thus occupying the region above the dashed boundary line in Figure 9 (left), while those with $\lambda_{\mathrm{e}}<0.31 \times \sqrt{\epsilon}$, which lie below this boundary line, are slow rotators. We note that there are "intermediate-type" galaxies with $\lambda_{\mathrm{e}}=0.31 \times \sqrt{\epsilon}$. Figure 9 clearly reveals that NGC 7025's pseudo-bulge is a fast rotator.

In addition, given the high-resolution data afforded by MEGARA, we attempted to characterize the rotation of the pseudo-bulge as a function of its radius. Figure 9 (right) plots spin tracks for the HR-I and LR-V data created by measuring $\lambda(R)$ and $\epsilon(R)$ within nine different circular apertures with radii $R=(0.07,0.18,0.29,0.41,0.53,0.64,0.76,0.87$, and $1.00) \times R_{\mathrm{e}}$. These tracks reveal that the pseudo-bulge, which has outwardly rising $\lambda(R)$ and $\epsilon(R)$ for $0.3 \lesssim R / R_{\mathrm{e}} \lesssim 1.0$, is consistent with being a fast rotator for all the apertures we considered. It is worth noting that the spin tracks by Graham et al. (2017, their Figure 9) differ from ours. Instead of using circular apertures as done here, they suggested determining $\lambda$ $(R)$ within elliptical annuli to better represent the rotation profiles for their galaxies with intermediate-scale disks (see also Bellstedt et al. 2017). Given that we are interested in determining the radial $\lambda(R)$ profiles only for NGC 7025's pseudo-bulge component, $\lambda$ values measured using circular apertures are more meaningful than those determined within elliptical annuli.

\section{Discussion}

\subsection{The Nature of the Disky Bulge in NGC 7025}

High-resolution MEGARA spectroscopy of the early-type spiral (Sa) galaxy NGC 7025, obtained as part of the commissioning run of the MEGARA instrument, reveals a disky bulge that is supported by rotation. As noted in the preceding sections, this bulge is well fitted by a Sérsic model with a low Sérsic index $(n \sim 1.5-1.9)$. Its properties are
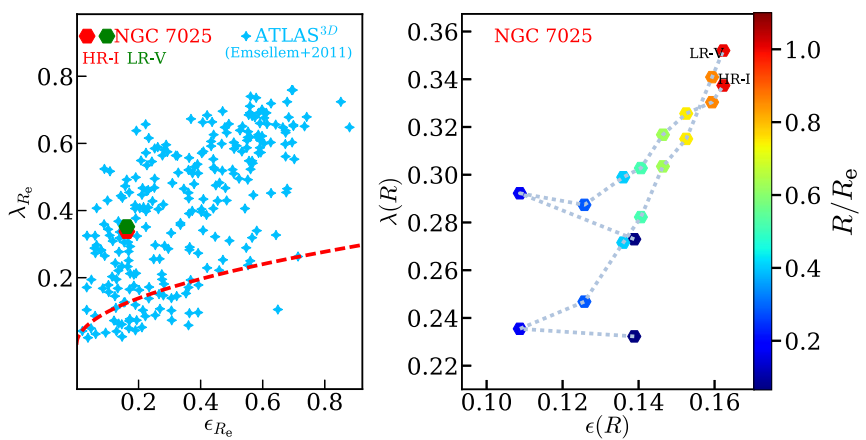

Figure 9. Left panel: division of galaxies into fast and slow rotators in the spin parameter $\lambda_{\mathrm{e}}$ vs. ellipticity $\epsilon_{\mathrm{e}}$ diagram. Fast (slow) rotators are located above (below) the dashed demarcation line $\left(\lambda_{\mathrm{e}}=0.31 \times \sqrt{\epsilon}_{\mathrm{e}}\right)$ adopted by Emsellem et al. (2011). For NGC 7025, we show two $\lambda_{\mathrm{e}}$ values derived based on the pPXF best fits to the galaxy's HR-I and LR-V spectra (Figures 1 and 2). Right panel: trails of $\lambda(R)$ vs. $\epsilon(R)$ for NGC 7025 showing outwardly rising $\lambda$ and $\epsilon$ at $0.3 \lesssim R / R_{\mathrm{e}} \lesssim 1.0$

generally consistent with a secular-driven formation scenario (e.g., Kormendy 1982, 1993; Pfenniger 1993; Courteau et al. 1996; Carollo et al. 1997; Athanassoula 2005; Laurikainen et al. 2007; Fisher \& Drory 2008), as such favoring a pseudobulge interpretation. In contrast, Neumann et al. (2017) and Gilhuly \& Courteau (2018) advocated a classical bulge for the galaxy. The 2D decompositions of the SDSS images of NGC 7025 by Neumann et al. (2017) and Gilhuly \& Courteau (2018) were performed without accounting for the galaxy intermediate (spiral-arm) component (see also Rizzo et al. 2018). This biased their Sérsic index and flux for the bulge component toward higher values, which are expected for classical bulges (see Section 3.2.1).

As mentioned in the introduction, in the secular evolution picture, pseudo-bulges are thought to have formed (slowly) via the inward funneling of disk materials facilitated by nonaxisymmetric features, such as bars, ovals, lenses, or spiral arms (e.g., Pfenniger \& Norman 1990; Kormendy 1993; Kormendy \& Kennicutt 2004; Laurikainen et al. 2007; Fisher \& Drory 2008; Graham \& Worley 2008; Laurikainen et al. 2010; Méndez-Abreu et al. 2014; Erwin et al. 2015; Tonini et al. 2016). Therefore, the prediction is that there is coupling between the pseudo-bulges and their surrounding large-scale disks as revealed, for example, by the nearly constant bulge-todisk size ratios $\left(R_{\mathrm{e}} / h\right)$ of $\sim 0.20$ and 0.24 for late- and earlytype spirals, respectively (MacArthur et al. 2003; see also Courteau et al. 1996; Graham 2001; Fisher \& Drory 2008; Graham \& Worley 2008; Gadotti 2009). Laurikainen et al. (2010) found median $R_{\mathrm{e}} / h$ values of 0.15 and 0.20 for their S0a and S0 galaxies. For NGC 7025, we found $R_{\mathrm{e}} / h \sim 0.18-0.20$ (Table 1). Given that MacArthur et al. (2003) did not account for spiral arms in their light profile decompositions, our $R_{\mathrm{e}} / h$ value for NGC 7025 is generally in good agreement with those of early-type spiral and S0 galaxies (MacArthur et al. 2003; Laurikainen et al. 2010).

NGC 7025's pseudo-bulge fulfills three pseudo-bulge identification criteria by Kormendy \& Kennicutt (2004):

1. Having rotationally supported dynamics (Figures 7-9)

2. Having a nearly exponential light profile (Figures 3 and 4 and Tables 1 and 3) and dust and stellar spiral structures which extend all the way into the galaxy center (Figures 5 and 6) 
3. Furthermore, our $n$ values for the pseudo-bulge agree with the Fisher \& Drory (2008) pseudo-bulge $(n \lesssim 2)$ versus classical bulge $(n \gtrsim 2)$ divide (see also Neumann et al. 2017, who advocated a similar $n$-based pseudobulge/classical bulge dichotomy but using a threshold $n=1.5$ instead of 2). However, we refer the reader to a detailed discussion by Graham (2013, see his Section 4.3), who highlighted the danger of using such bulge classification criteria alone.

Our pseudo-bulge-to-total flux ratio for NGC 7025 $(B / T \sim 0.28-30)$ is in excellent agreement with Erwin et al. (2015), who reported a mean $B / T$ of $\sim 0.33$ for his sample of pseudo-bulges (see also Fisher \& Drory 2008, their Figure 11), and is akin to the $B / T$ values of $\sim 0.25-0.35$ typically reported for other spiral and lenticular galaxies (see, e.g., Fisher \& Drory 2008; Graham \& Worley 2008; Gadotti 2009; Weinzirl et al. 2009; Laurikainen et al. 2010; Dullo et al. 2016, references therein).

Pseudo-bulges and their surrounding disks are expected to have similar colors, relatively bluer than those of classical bulges built in violent merging events (e.g., Peletier \& Balcells 1996; Carollo et al. 1997; Fisher \& Drory 2008; Driver et al. 2007; Cameron et al. 2009; Gadotti 2009). Excluding the most PSF-affected region $\left(R \lesssim 1^{\prime \prime}\right)$ and going out from the center, the $g-i$ color profile of NGC 7025 plotted in Figure 3 becomes gradually bluer; it then levels at intermediate radii $\left(\sim 10^{\prime \prime} \lesssim R \lesssim 40^{\prime \prime}\right)$ before turning redder at large radii where the disk dominates. Not only does the galaxy color profile agree with the secular evolution scenario, it also resembles the "U-shaped" color profile of "Type II" galaxies (Bakos et al. 2008, their Figure 1), having truncated exponential profiles believed to arise from disk instabilities driven by bars (e.g., Pohlen \& Trujillo 2006; Borlaff et al. 2016; Catalán-Torrecilla et al. 2017; Bouquin et al. 2018). However, the $g-i$ color of NGC $7025(\sim 1.49 \pm 0.11$; see Figure 5$)$ is markedly redder than the typical $g-i=1.06 \pm 0.17$ color reported for $\mathrm{Sa}$ galaxies (Shimasaku et al. 2001, their Table 1). We have converted their $g^{*}-i^{*}$ colors into $g-i$ using the SDSS DR7 photometric equations. ${ }^{17}$ The presence of dust may partly explain the redder color of the galaxy.

\subsection{Formation of NGC 7025's Pseudo-bulge}

Within the secular evolution formation scenario, massive pseudo-bulges are closely linked to strong bars, owing to the high efficiency of such bars at funneling disk materials toward the inner regions of galaxies (e.g., Kormendy \& Kennicutt 2004; Durbala et al. 2008; Cheung et al. 2013). Although NGC 7025 does not contain a bar or an oval structure, its compact $\left(R_{\mathrm{e}} \sim 1.48-2.06 \mathrm{kpc}\right)$ pseudo-bulge is massive, i.e., a stellar mass $M_{* \text {,bulge }} \sim(4.21-4.47) \times 10^{10} M_{\odot}$, only a factor of 1.66-3.33 less massive than the 21 local compact $\left(R_{\mathrm{e}} \lesssim 2 \mathrm{kpc}\right)$ and massive $\left(0.7 \times 10^{11} M_{\odot}<M_{*}<1.4 \times 10^{11} M_{\odot}\right)$ bulges identified by Graham et al. (2015). The latter bulges were shown to have physical properties similar to those of the compact, massive high-redshift galaxies found at $z \sim 1.5-2$ (Dullo \& Graham 2013; Graham 2013; Dullo 2014; Graham et al. 2015; de la Rosa et al. 2016). We tentatively propose that a secular process involving the tightly wound stellar spiral arms of NGC 7025 may be driving gas and stars out of the disk into

\footnotetext{
${ }^{17}$ http://classic.sdss.org/dr7/algorithms/fluxcal.html
}

the inner regions of the galaxy, building up its massive pseudobulge (e.g., Carollo et al. 1997; Kormendy \& Kennicutt 2004; Laurikainen et al. 2007). Indeed, NGC 7025 is the only unbarred isolated galaxy from the sample of 49 CALIFA galaxies by Holmes et al. (2015) which exhibits noncircular, barlike flows. Simulations have shown bar dissolution caused by central mass concentrations (e.g., Norman et al. 1996; Shen \& Sellwood 2004; Athanassoula et al. 2005), but this process is not well understood. Given the low concentration of the light distribution of NGC 7025's pseudo-bulge as revealed by the low $n$ value, it seems unlikely that the galaxy had a bar in the past that was destroyed by the above process.

\subsection{Alternative Pseudo-bulge Formation Scenarios}

Alternative pseudo-bulge formation scenarios have been discussed in the literature. For example, simulations have shown pseudo-bulges built via high-redshift starbursts with minor contributions from secular evolution (Okamoto 2013) and through gas-rich minor and/or major galaxy merger events (e.g., Springel \& Hernquist 2005; Eliche-Moral et al. 2011; Guedes et al. 2013; Querejeta et al. 2015; Athanassoula et al. 2016; Tonini et al. 2016; Sauvaget et al. 2018). In the former scenario, the starburst-built pseudo-bulges form before their surrounding disks, and they are already in place at redshifts of $z \sim 2-3$. This is similar to the high-redshift clump pseudobulge formation scenario by Inoue \& Saitoh (2012), but both these high-redshift scenarios are inconsistent with (i) the simulations by Elmegreen et al. (2008), which revealed that the coalescence of high-redshift clumps due to disk instabilities leads to the formation of bulges that resemble classical bulges, and with (ii) the color profile of NGC 7025 mentioned above (see Figure 3). For the merger-driven scenarios, the pseudobulges tend to have young stellar populations (except for those by Guedes et al. 2013), and they are formed prior to their surrounding disks. However, NGC 7025 is an isolated spiral galaxy; its low-density environment does not favor the mergerdriven scenario. Indeed, $\sim 88 \%$ of the sample of isolated galaxies studied by Hirschmann et al. (2013) have experienced at most one minor merger over their lifetime (see also Durbala et al. 2008). Also, Martig et al. (2012) reported that most of their isolated spiral galaxies with $B / T<0.3$ experienced only minor mergers since $z \sim 2$. Using theoretical galaxy formation models, Tonini et al. (2016, their Figure 9) also reported that bulges built through secular evolution dominate the galaxy mass distribution at intermediate masses, $10 \lesssim \log \left(M_{*} / M_{\odot}\right) \lesssim 11 ;$ in contrast, merger-built bulges dominate at the lower- and higher-mass ends (see also Martig et al. 2012).

Furthermore, it has been shown that galaxy mergers or accretion of external gas can produce kinematically distinct (counterrotating) stellar components in galaxies (e.g., Kormendy 1984; Balcells \& Quinn 1990; Bertola et al. 1992; Kannappan \& Fabricant 2001; Krajnović et al. 2011; Dullo et al. 2018, their Section 4.3.2). Krajnović et al. (2011) identified such kinematic features in roughly $26 \%$ of their ATLAS $^{3 \mathrm{D}}$ sample of 260 early-type galaxies. The absence of any significant peculiarity in the stellar kinematics of NGC 7025 (Figure 7 and Falcón-Barroso et al. 2017) provides further support for the secular evolution scenario, but this is not to say that merger events cannot build bulges with featureless kinematics. 


\section{Conclusions}

This paper presents the first data and scientific results of MEGARA, a high-resolution IFU and MOS installed on the GTC. We carried out MEGARA IFU observations of the Sa galaxy NGC 7025. The results from these observations show that MEGARA is able to deliver high-resolution IFU data ideal for studying the central kinematic properties of nearby galaxies. We also performed detailed $1 \mathrm{D}$ and $2 \mathrm{D}$ decompositions of this galaxy's SDSS $i$-band data into a Gaussian $(n=0.5)$ nuclear component, a Sérsic bulge, a Sérsic intermediate-scale spiralarm component, and an outer exponential $(n=1)$ disk. The main results from this work are as follows.

(1) We found that NGC 7025's bulge has a Sérsic index $n \sim 1.80 \pm 0.24$, a half-light radius $R_{\mathrm{e}} \sim 1.70 \pm 0.43$ kpc, stellar mass $M_{*} \sim(4.34 \pm 1.70) \times 10^{10} M_{\odot}$, and a bulge-to-total flux ratio $B / T \sim 0.30$.

(2) We have presented the spins $(\lambda)$ and ellipticities $(\epsilon)$ of the bulge enclosed within nine different circular apertures with radii $R \leqslant R_{\mathrm{e}}$, revealing that the bulge, which exhibits a spin track of outwardly rising $\lambda$ and $\epsilon$, is a fast rotator for all the apertures considered. Correcting the bulge's stellar velocity $V$ for inclination, we constructed $V_{\text {corr }} / \sigma$ maps, which show that the bulge is mainly supported by rotation.

(3) The photometric and kinematic findings given previously strongly favor a pseudo-bulge in NGC 7025, in contrast to past works, which have advocated a classical bulge interpretation.

(4) Our results for NGC 7025 are broadly consistent with the secular evolution model of pseudo-bulge formation. The disky pseudo-bulge of the galaxy may have formed naturally from the outer disk, slowly driven by the tightly wound stellar spiral arms. Examination of the stellar kinematics and population together with a careful analysis of high-resolution HST images for a large number of unbarred late-type galaxies is desirable to check how frequent massive $\left(M_{*} \sim 5 \times 10^{10} M_{\odot}\right)$ pseudo-bulges can be built up by secular evolution with spiral arms as the driving agents.

We thank the referee for their careful reading of the paper and many suggestions that improved the paper. B.T.D. acknowledges support from a Spanish postdoctoral fellowship, "Ayudas 1265 para la atracción del talento investigador. Modalidad 2: jóvenes investigadores," funded by Comunidad de Madrid under grant no. 2016-T2/TIC-2039. We acknowledge financial support from the Spanish Ministry of Economy and Competitiveness under grant no. AYA2016-75808-R, which is partly funded by the European Regional Development Fund, and from the Excellence Network MægNet (AYA2017-90589-REDT). We acknowledge financial support from SpaceTec-CM: Desarrollo de nuevas tecnologías para instrumentación espacial en la Comunidad de Madrid, S2013/ICE-2822.

Funding for SDSS-IV has been provided by the Alfred P. Sloan Foundation, the U.S. Department of Energy Office of Science, and the Participating Institutions. SDSS-IV acknowledges support and resources from the Center for HighPerformance Computing at the University of Utah. The SDSS website is located at www.sdss.org.

SDSS-IV is managed by the Astrophysical Research Consortium for the Participating Institutions of the SDSS
Collaboration, which include the Brazilian Participation Group, the Carnegie Institution for Science, Carnegie Mellon University, the Chilean Participation Group, the French Participation Group, the Harvard-Smithsonian Center for Astrophysics, Instituto de Astrofísica de Canarias, Johns Hopkins University, the Kavli Institute for the Physics and Mathematics of the Universe/University of Tokyo, Lawrence Berkeley National Laboratory, Leibniz Institut für Astrophysik Potsdam, MaxPlanck-Institut für Astronomie (Heidelberg), Max-PlanckInstitut für Astrophysik (Garching), Max-Planck-Institut für Extraterrestrische Physik, the National Astronomical Observatories of China, New Mexico State University, New York University, University of Notre Dame, Observatário Nacional/ MCTI, Ohio State University, Pennsylvania State University, Shanghai Astronomical Observatory, the United Kingdom Participation Group, Universidad Nacional Autónoma de México, University of Arizona, University of Colorado Boulder, University of Oxford, University of Portsmouth, University of Utah, University of Virginia, University of Washington, University of Wisconsin, Vanderbilt University, and Yale University.

\section{ORCID iDs}

Bililign T. Dullo (i) https://orcid.org/0000-0002-4140-0110 Armando Gil de Paz (1) https://orcid.org/0000-00016150-2854 África Castillo-Morales (1) https://orcid.org/0000-00034964-3245

Jesús Gallego (1) https://orcid.org/0000-0003-1439-7697 Jorge Iglesias-Páramo (i) https://orcid.org/0000-00032726-6370

Sergio Pascual ำ https://orcid.org/0000-0002-9351-6051 Nicolás Cardiel @ic https://orcid.org/0000-0002-9334-2979 Cristina Catalán-Torrecilla (ib https://orcid.org/0000-00028067-0164

\section{References}

Allen, P. D., Driver, S. P., Graham, A. W., et al. 2006, MNRAS, 371, 2 Athanassoula, E. 2005, MNRAS, 358, 1477

Athanassoula, E., Lambert, J. C., \& Dehnen, W. 2005, MNRAS, 363, 496 Athanassoula, E., Rodionov, S. A., Peschken, N., \& Lambert, J. C. 2016, ApJ, 821,90

Bakos, J., Trujillo, I., \& Pohlen, M. 2008, ApJL, 683, L103

Balcells, M., \& Quinn, P. J. 1990, ApJ, 361, 381

Barnes, J. E. 1988, ApJ, 331, 699

Bellstedt, S., Graham, A. W., Forbes, D. A., et al. 2017, MNRAS, 470, 1321 Bender, R., Saglia, R. P., \& Gerhard, O. E. 1994, MNRAS, 269, 785

Bender, R., Surma, P., Doebereiner, S., Moellenhoff, C., \& Madejsky, R. 1989, A\&A, 217, 35

Bertin, E., \& Arnouts, S. 1996, A\&AS, 117, 393

Bertola, F., Buson, L. M., \& Zeilinger, W. W. 1992, ApJL, 401, L79

Böker, T., Stanek, R., \& van der Marel, R. P. 2003, AJ, 125, 1073

Borlaff, A., Eliche-Moral, M. C., Beckman, J., \& Font, J. 2016, A\&A, 591, L7

Bouquin, A. Y. K., Gil de Paz, A., Muñoz-Mateos, J. C., et al. 2018, ApJS, 234, 18

Bureau, M., \& Freeman, K. C. 1999, AJ, 118, 126

Buta, R. J., Sheth, K., Athanassoula, E., et al. 2015, ApJS, 217, 32

Cameron, E., Driver, S. P., Graham, A. W., \& Liske, J. 2009, ApJ, 699, 105 Cappellari, M. 2017, MNRAS, 466, 798

Cappellari, M., \& Copin, Y. 2003, MNRAS, 342, 345

Cappellari, M., \& Emsellem, E. 2004, PASP, 116, 138

Carollo, C. M., Stiavelli, M., de Zeeuw, P. T., \& Mack, J. 1997, AJ, 114, 2366 Catalán-Torrecilla, C., Gil de Paz, A., Castillo-Morales, A., et al. 2017, ApJ, 848,87

Cheung, E., Athanassoula, E., Masters, K. L., et al. 2013, ApJ, 779, 162

Combes, F., Debbasch, F., Friedli, D., \& Pfenniger, D. 1990, A\&A, 233, 82 Courteau, S., de Jong, R. S., \& Broeils, A. H. 1996, ApJL, 457, L73 
Davies, R. L., Efstathiou, G., Fall, S. M., Illingworth, G., \& Schechter, P. L. 1983, ApJ, 266, 41

de Jong, R. S. 1996, A\&AS, 118, 557

de la Rosa, I. G., La Barbera, F., Ferreras, I., et al. 2016, MNRAS, 457, 1916 de Vaucouleurs, G. 1948, AnAp, 11, 247

de Vaucouleurs, G. 1963, ApJS, 8, 31

de Vaucouleurs, G., de Vaucouleurs, A., Corwin, H. G., Jr., et al. 1991, Third Reference Catalogue of Bright Galaxies. Volume I: Explanations and references. Volume II: Data for galaxies between $0 \mathrm{~h}$ and $12^{\mathrm{h}}$. Volume III: Data for galaxies between $12^{\mathrm{h}}$ and $24^{\mathrm{h}}$.

Driver, S. P., Allen, P. D., Liske, J., \& Graham, A. W. 2007, ApJL, 657, L85

Driver, S. P., Popescu, C. C., Tuffs, R. J., et al. 2008, ApJL, 678, L101

Dullo, B. T. 2014, in ASP Conf. Ser. 480, Structure and Dynamics of Disk Galaxies, ed. M. S. Seigar \& P. Treuthardt (San Francisco, CA: ASP), 75

Dullo, B. T., \& Graham, A. W. 2012, ApJ, 755, 163

Dullo, B. T., \& Graham, A. W. 2013, ApJ, 768, 36

Dullo, B. T., \& Graham, A. W. 2014, MNRAS, 444, 2700

Dullo, B. T., \& Graham, A. W. 2015, ApJ, 798, 55

Dullo, B. T., Graham, A. W., \& Knapen, J. H. 2017, MNRAS, 471, 2321

Dullo, B. T., Knapen, J. H., Williams, D. R. A., et al. 2018, MNRAS, 475, 4670

Dullo, B. T., Martínez-Lombilla, C., \& Knapen, J. H. 2016, MNRAS, 462, 3800

Durbala, A., Sulentic, J. W., Buta, R., \& Verdes-Montenegro, L. 2008, MNRAS, 390, 881

Dutton, A. A., Treu, T., Brewer, B. J., et al. 2013, MNRAS, 428, 3183

Eggen, O. J., Lynden-Bell, D., \& Sandage, A. R. 1962, ApJ, 136, 748

Eliche-Moral, M. C., González-García, A. C., Balcells, M., et al. 2011, A\&A, 533, A104

Elmegreen, B. G., Bournaud, F., \& Elmegreen, D. M. 2008, ApJ, 688, 67

Emsellem, E., Cappellari, M., Krajnović, D., et al. 2007, MNRAS, 379, 401

Emsellem, E., Cappellari, M., Krajnović, D., et al. 2011, MNRAS, 414, 888

Erwin, P. 2015, ApJ, 799, 226

Erwin, P., Saglia, R. P., Fabricius, M., et al. 2015, MNRAS, 446, 4039

Erwin, P. 2018, MNRAS, 474, 5372

Erwin, P., Beltrán, J. C. V., Graham, A. W., \& Beckman, J. E. 2003, ApJ, 597, 929

Faber, S. M., Tremaine, S., Ajhar, E. A., et al. 1997, AJ, 114, 1771

Fabricius, M. H., Saglia, R. P., Fisher, D. B., et al. 2012, ApJ, 754, 67

Falcón-Barroso, J., Lyubenova, M., van de Ven, G., et al. 2017, A\&A, 597, A48

Fisher, D. B., \& Drory, N. 2008, AJ, 136, 773

Franx, M., Illingworth, G., \& de Zeeuw, T. 1991, ApJ, 383, 112

Gadotti, D. A. 2008, MNRAS, 384, 420

Gadotti, D. A. 2009, MNRAS, 393, 1531

Gavazzi, G., Franzetti, P., Scodeggio, M., Boselli, A., \& Pierini, D. 2000, A\&A, 361, 863

Gil de Paz, A., Carrasco, E., Gallego, J., et al. 2016, Proc. SPIE, 9908, 99081K Gilhuly, C., \& Courteau, S. 2018, MNRAS, 477, 845

González-García, A. C., Balcells, M., \& Olshevsky, V. S. 2006, MNRAS, 372, L78

Graham, A. W. 2001, AJ, 121, 820

Graham, A. W. 2013, in Planets, Stars and Stellar Systems, vol. 6, ed. T. D. Oswalt \& W. C. Keel, 91

Graham, A. W., Ciambur, B. C., \& Savorgnan, G. A. D. 2016, ApJ, 831, 132

Graham, A. W., Dullo, B. T., \& Savorgnan, G. A. D. 2015, ApJ, 804, 32

Graham, A. W., Janz, J., Penny, S. J., et al. 2017, ApJ, 840, 68

Graham, A. W., Spitler, L. R., Forbes, D. A., et al. 2012, ApJ, 750, 121

Graham, A. W., \& Worley, C. C. 2008, MNRAS, 388, 1708

Guedes, J., Mayer, L., Carollo, M., \& Madau, P. 2013, ApJ, 772, 36

Hirschmann, M., De Lucia, G., Iovino, A., \& Cucciati, O. 2013, MNRAS, 433, 1479

Holmes, L., Spekkens, K., Sánchez, S. F., et al. 2015, MNRAS, 451, 4397

Illingworth, G. 1977, ApJL, 218, L43
Inoue, S., \& Saitoh, T. R. 2012, MNRAS, 422, 1902

Into, T., \& Portinari, L. 2013, MNRAS, 430, 2715

Jedrzejewski, R. I. 1987, MNRAS, 226, 747

Kannappan, S. J., \& Fabricant, D. G. 2001, AJ, 121, 140

Kauffmann, G. 1996, MNRAS, 281, 487

Knapen, J. H., Shlosman, I., Heller, C. H., et al. 2000, ApJ, 528, 219

Kormendy, J. 1982, ApJ, 257, 75

Kormendy, J. 1984, ApJ, 287, 577

Kormendy, J. 1993, in IAU Symp. 153, Galactic Bulges, ed. H. Dejonghe \& H. J. Habing (Dordrecht: Kluwer), 209

Kormendy, J. 2013, in Canary Islands Winter School of Astrophysics, Secular Evolution of Galaxies, ed. J. Falcón-Barroso \& J. H. Knapen (Cambridge: Cambridge University Press), 1

Kormendy, J. 2016, in Galactic Bulges, ed. E. Laurikainen, R. Peletier, \& D. Gadotti (New York: Springer), 431

Kormendy, J., \& Illingworth, G. 1982, ApJ, 256, 460

Kormendy, J., \& Kennicutt, R. C., Jr. 2004, ARA\&A, 42, 603

Krajnović, D., Bacon, R., Cappellari, M., et al. 2008, MNRAS, 390, 93

Krajnović, D., Emsellem, E., Cappellari, M., et al. 2011, MNRAS, 414, 2923

Kuijken, K., \& Merrifield, M. R. 1995, ApJL, 443, L13

Laurikainen, E., \& Salo, H. 2016, in Galactic Bulges, vol. 418, ed. E. Laurikainen, R. Peletier, \& D. Gadotti (Berlin: Springer), 77

Laurikainen, E., Salo, H., \& Buta, R. 2005, MNRAS, 362, 1319

Laurikainen, E., Salo, H., Buta, R., \& Knapen, J. H. 2007, MNRAS, 381, 401

Laurikainen, E., Salo, H., Buta, R., Knapen, J. H., \& Comerón, S. 2010 MNRAS, 405, 1089

MacArthur, L. A., Courteau, S., \& Holtzman, J. A. 2003, ApJ, 582, 689

Martig, M., Bournaud, F., Croton, D. J., Dekel, A., \& Teyssier, R. 2012, ApJ, 756, 26

Martinez-Valpuesta, I., Shlosman, I., \& Heller, C. 2006, ApJ, 637, 214

Méndez-Abreu, J., Debattista, V. P., Corsini, E. M., \& Aguerri, J. A. L. 2014, A\&A, 572, A25

Neumann, J., Wisotzki, L., Choudhury, O. S., et al. 2017, A\&A, 604, A30

Norman, C. A., Sellwood, J. A., \& Hasan, H. 1996, ApJ, 462, 114

Okamoto, T. 2013, MNRAS, 428, 718

Paturel, G., Petit, C., Prugniel, P., et al. 2003, A\&A, 412, 45

Peletier, R. F. 2008, in ASP Conf. Ser. 390, Pathways Through an Eclectic Universe, ed. J. H. Knapen, T. J. Mahoney, \& A. Vazdekis (San Francisco, CA: ASP), 232

Peletier, R. F., \& Balcells, M. 1996, AJ, 111, 2238

Peng, C. Y., Ho, L. C., Impey, C. D., \& Rix, H.-W. 2010, AJ, 139, 2097

Pfenniger, D. 1993, in IAU Symp. 153, Galactic Bulges, ed. H. Dejonghe \& H. J. Habing (Dordrecht: Kluwer), 387

Pfenniger, D., \& Norman, C. 1990, ApJ, 363, 391

Pohlen, M., \& Trujillo, I. 2006, A\&A, 454, 759

Querejeta, M., Eliche-Moral, M. C., Tapia, T., et al. 2015, A\&A, 579, L2

Rizzo, F., Fraternali, F., \& Iorio, G. 2018, MNRAS, 476, 2137

Salpeter, E. E. 1955, ApJ, 121, 161

Sauvaget, T., Hammer, F., Puech, M., et al. 2018, MNRAS, 473, 2521

Sérsic, J. L. 1968, Atlas de Galaxias Australes (Cordoba, Argentina: Observatorio Astronomico)

Shen, J., \& Sellwood, J. A. 2004, ApJ, 604, 614

Sheth, K., Elmegreen, D. M., Elmegreen, B. G., et al. 2008, ApJ, 675, 1141

Shimasaku, K., Fukugita, M., Doi, M., et al. 2001, AJ, 122, 1238

Springel, V., \& Hernquist, L. 2005, ApJL, 622, L9

Tonini, C., Mutch, S. J., Croton, D. J., \& Wyithe, J. S. B. 2016, MNRAS 459, 4109

van de Sande, J., Bland-Hawthorn, J., Fogarty, L. M. R., et al. 2017, ApJ, 835,104

Vazdekis, A., Koleva, M., Ricciardelli, E., Röck, B., \& Falcón-Barroso, J. 2016, MNRAS, 463, 3409

Weinzirl, T., Jogee, S., Khochfar, S., Burkert, A., \& Kormendy, J. 2009, ApJ, 696, 411 